\title{
Análise da influência da malha de pontos em índices de avaliação de desempenho da luz natural
}

Analysis of grid of points influence on daylight metrics

\section{Pedro Vitor Sousa Ribeiro Ricardo Carvalho Cabús}

\section{Resumo}

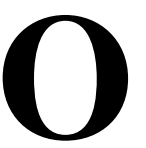

desempenho da iluminação natural de ambientes pode ser avaliado por meio de várias ferramentas, a maioria baseada na iluminância. Mesmo com os avanços nas últimas décadas, principalmente os computacionais, a definição dos pontos da malha é deixada, em geral, para escolha do simulador. Este artigo tem como objetivo analisar a influência da malha de pontos no desempenho das métricas: iluminância média (Em), uniformidade (U), autonomia de luz natural (ALN), autonomia de luz natural contínua (ALNc), autonomia de luz natural espacial (ALNe), iluminância útil de luz natural (IULN) e exposição solar anual (ESA). A simulação computacional foi realizada pelo TropLux, em ambiente de $6 \mathrm{~m}^{2} \times 6 \mathrm{~m}^{2}$ sem proteção solar. Realizouse análise exploratória e estatística, utilizando a ANOVA, teste de Tukey e matriz de correlação. Os resultados indicam que a grade de pontos influencia na precisão das métricas estudadas. Para Em, U, ALN, ALNc, ALNe e IULN os resultados se estabilizaram a partir de uma malha de $6 \times 6$ pontos, com distância entre pontos de 1,00 m, enquanto para ESA ocorreu a partir da malha de 11x11 pontos, ou distância entre pontos de $0,54 \mathrm{~m}$. O trabalho sugere que uma análise distinta das contribuições da luz do sol direta e da luz do céu levando em consideração o tamanho da malha pode otimizar o desempenho computacional, mantendo a precisão.

Palavras-chave: Iluminação natural. Malha de pontos. Índices de desempenho da luz natural. Simulação computacional.

\begin{abstract}
The luminous performance of buildings can be evaluated using several tools, most of them based on illuminance. Although progress has been made in daylight metrics in recent decades, mainly due to developments in computer systems technology, they do not usually address how grids are defined. This paper analyses the influence of the grid influence on the performance of the following metrics: average illuminance (Em), uniformity (U), daylight autonomy (DA), continuous daylight autonomy ( $C D A)$, spatial daylight autonomy (sDA), useful daylight illuminance (UDI) and annual solar exposure (ASE). Daylight simulations were done using TropLux, in a $6 \times 6 \mathrm{~m}^{2}$ room without shading device. Exploratory and statistical analyses were

performed using the ANOVA method, Tukey tests and a correlation matrix. The results indicate that the grid of points influences on the precision of the studied metrics. For Em, $U, D A, C D A, s D A$ and UDI, the results stabilised starting from a $6 \times 6$ grid with a 1,00m distance between points. For ASE, a grid with $11 \times 11$ points, or a $0.54 \mathrm{~m}$ distance between points was required. This study suggests that a different analysis of skylight and direct sunlight, taking into account grid size, may optimise computer performance maintaining accuracy.
\end{abstract}

1 Pedro Vitor Sousa Ribeiro ${ }^{1}$ Universidade Federal de Alagoas Maceió - AL - Brasil https:// orcid. org/ 0000-0003-0152-4319

${ }^{2}$ Ricardo Carvalho Cabús ${ }^{2}$ Universidade Federal de Alagoas Maceió - AL - Brasil

Recebido em 19/11/18 Aceito em 07/03/19
Keywords: Daylighting. Grid of points. Daylight metrics. Computer simulation. 


\section{Introdução}

A avaliação do desempenho luminoso de um ambiente pode ser feita utilizando diversas ferramentas como simulação computacional ou por meio de medição in loco. O avanço na velocidade de processamento e a facilidade de utilização e acesso aos computadores pessoais têm determinado o aumento da frequência de uso dessa ferramenta, no estudo da iluminação natural de edificações. Definidos os parâmetros de processamento e a geometria do modelo de estudo, os softwares de simulação em iluminação natural fornecem resultados por imagens ou por dados numéricos.

Alguns índices podem ser utilizados para determinar a qualidade da iluminação natural dos ambientes. A maioria deles utiliza, em seu cálculo, a iluminância como valor-base. O trabalho utiliza os índices apenas como ferramenta de comparação, não sendo eles, ou a sua forma de cálculo, objeto de estudo. Os índices são detalhados a seguir.

\section{Iluminância média (Em)}

A iluminância é a ferramenta mais simples de análise do desempenho luminoso de um ambiente. A norma brasileira NBR 15215: Iluminação Natural (ANBT, 2005a, 2005b, 2005c) utiliza a iluminância como parâmetro de avaliação da disponibilidade de luz natural em ambientes construídos. Aponta, ainda, os procedimentos para sua medição in loco ou por meio de simulação, utilizando um método gráfico.

Outra norma que prevê a determinação da iluminância é a NBR 15575: Edificações Habitacionais - Desempenho (ABNT, 2013a), por meio de simulação ou de medições in loco. Entretanto, Guidi et al. (2018) observam que o procedimento de cálculo para a determinação da iluminação natural descrito não configura uma simulação computacional, e sim um método prescritivo. Normas de iluminação artificial, como a ISO/CIE 8995-1: Iluminação de Ambientes de Trabalho (ABNT, 2013b), adotam esse índice como parâmetro referência para a avaliação de desempenho luminoso de ambientes internos. No trabalho foi usada a iluminância média do plano de trabalho de forma a simplificar a análise comparativa.

\section{Uniformidade da iluminância (U)}

A CIBSE (CHARTERED..., 1999) aponta que o uso da iluminância média como único parâmetro de avaliação não é efetivo para a verificação da qualidade da iluminação, sendo necessária a utilização de outros instrumentos, como a uniformidade. Alguns relatórios técnicos, como a
CEC-500-06-039 (CALIFORNIA..., 2012), e alguns autores (LITTLEFAIR; AIZLEWOOD; BIRTLES, 1994; RAKHA; NASSAR, 2011; MANGKUTO; ROHMAH; ASRI, 2016) afirmam que o conceito de uniformidade pode ser ampliado para utilização em iluminação natural.

A uniformidade da iluminância é a razão entre seu valor mínimo e a média no plano de trabalho (ABNT, 2013b). Autores como Baker, Fanchiotti e Steemers (1993) apontam-na como uma das componentes indispensáveis na avaliação da qualidade da iluminação natural. Phillips (2004) afirma que o aumento da uniformidade deve anteceder outras preocupações como a ocorrência de ofuscamento. Esse parâmetro permite avaliar a relação entre a quantidade de luz na área da tarefa e o seu entorno imediato, proporcionando melhoria na avaliação da iluminância, se comparado apenas ao valor médio global (SLATER; BOYCE, 1990). O ganho em uniformidade da iluminância está associado à utilização de dispositivos de controle da luz natural. Elementos como o brise e a prateleira de luz são utilizados com essa finalidade. No presente estudo não foram adotados tais elementos.

Autores como Slater e Boyce (1990) afirmam, para iluminação artificial, que a adoção do valor 0,7 de uniformidade no plano da tarefa é satisfatório em todas as atividades. Mangkuto, Rohmah e Asri (2016) aponta que para a adoção do conceito de uniformidade em iluminação natural faz-se necessário alterar o valor de referência, tendo em vista a variabilidade da fonte.

\section{Autonomia de Luz Natural (ALN)}

A ALN foi o primeiro parâmetro de avaliação que anualiza o estudo da iluminância. Desenvolvido pela Association Suissedes Electriciens (ASSOCIATION..., 1989) e aperfeiçoado por Reinhart e Walkenhorst (2001), é definida como o percentual do ano em que um requisito mínimo de iluminância é atingido somente por luz natural. Reinhart, Mardaljevic e Rogers (2006) adotam iluminância mínima de 500 lx, que pode ser alterada conforme a necessidade e o uso do ambiente em estudo.

Diversos autores propuseram índices derivados da ALN: autonomia de luz natural contínua (ALNc, ver Figura 1) (REINHART; MARDALJEVIC; ROGERS, 2006), autonomia de luz natural temporal (ALNt) (KLEINDIENST; ANDERSEN, 2012), autonomia de luz natural zonal (ILLUMINATING..., 2011) e autonomia de luz natural espacial (ALNe) (ILLUMINATING..., 2011). Em 2012 a Illuminating Engineering 
Society, tendo como base 61 pesquisas publicadas pela California Energy Commission (CALIFORNIA..., 2012), adotou como referencial a ALNe (ILLUMINATING..., 2012). A ALNe é o percentual de área do plano de trabalho em que a ALN é maior ou igual a 50\%. O normativo também aponta duas classificações possíveis: favorável ou preferível - quando o percentual de área é maior ou igual a $75 \%$-, e neutro ou aceitável - quando o percentual é maior que 55\%.

\section{Iluminância Útil de Luz Natural (IULN)}

A IULN, proposta por Nabil e Mardaljevic (2005), consiste em uma análise anualizada da iluminância do ambiente em um ponto, categorizando os valores obtidos em intervalos, conforme apresentados no Quadro 1. O conceito pode ser ampliado espacialmente para uma malha de pontos, resultando na IULN média do plano. Inicialmente, os autores adotaram o valor máximo de $2.000 \mathrm{~lx}$ (NABIL; MARDALJEVIC, 2005), ampliando posteriormente para $3.000 \mathrm{~lx}$ (MARDALJEVIC et al., 2011).

As faixas definidas pelo índice podem ser alteradas dependendo do uso do ambiente e dos requisitos de iluminação natural desejados. Os autores afirmam que esse conceito poderá substituir o tradicional fator de luz do dia (FLD) (NABIL; MARDALJEVIC, 2006).

\section{Exposição Solar Anual (ESA)}

A IES LM-83-12 (ILLUMINATING..., 2012) traz um indicador para avaliar a exposição à luz solar direta, a ESA. Ela avalia, de forma simplificada, o potencial de desconforto visual causado pela radiação solar direta em ambientes. O cálculo consiste em determinar a quantidade percentual de pontos ou de sensores em que a luz solar direta atinge mais de $1.000 \mathrm{~lx}$ em mais de 250 horas/ano (ILLUMINATING..., 2012). O ambiente deve ser avaliado ao longo de um ano, 10 horas por dia. Trabalhos recentes (PEREIRA, 2017; FERNANDEZ, 2017) que utilizam esse indicador comumente não discutem a forma como a quantidade e a disposição dos pontos de análise foram escolhidas.

Para aplicação do índice a IES LM-83-12 (ILLUMINATING..., 2012) prevê a utilização de protetor solar nas janelas, como brises ou persianas, entretanto permite que não sejam utilizados em casos nos quais não são previstos em projeto. Para o estudo não serão utilizados protetores solares nas aberturas.

\section{Malha de sensores e intervalo de tempo}

Nos estudos de iluminação natural em ambientes internos (GUIDI et al., 2018; CARVALHO, 2018; ALBUQUERQUE, AMORIM; 2012) observa-se a predominância da escolha da malha de pontos utilizando os parâmetros descritos na NBR 152154 (ABNT, 2005c) ou na ISO/CIE 8995-1 (INTERNATIONAL..., 2002). Em outros casos, os autores não informam a origem da escolha da malha (FONTENELLE; CLARO; ARAÚJO, 2011; MAIOLI; ALVAREZ, 2013) ou sequer informam quantos pontos foram utilizados (FONSECA; PEREIRA, 2017; FERNANDEZ, 2017; PEREIRA, 2017).

Figura 1 - Comparação entre os métodos de cálculo para ALN e ALNc

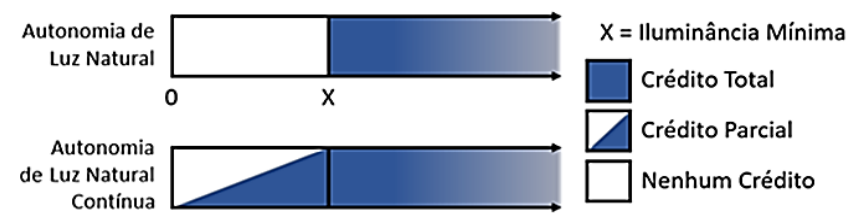

Quadro 1 - Zonas da iluminância útil de luz natural

\begin{tabular}{c|c|c}
\hline Intervalo & Classificação & A quantidade de luz natural é: \\
\hline $\mathrm{E}<100 \mathrm{~lx}$ & Não atinge a faixa útil & $\begin{array}{c}\text { Insuficiente como única fonte de iluminação, sendo } \\
\text { necessária uma significativa contribuição de } \\
\text { iluminação artificial. }\end{array}$ \\
\hline $100 \mathrm{~lx}<\mathrm{E}<500 \mathrm{~lx}$ & Atinge a faixa útil & $\begin{array}{c}\text { Efetiva, entretanto, ainda necessitará de } \\
\text { complementação artificial }\end{array}$ \\
\hline $500 \mathrm{~lx}<\mathrm{E}<3.000 \mathrm{~lx}$ & Atinge a faixa útil & Desejável \\
\hline $\mathrm{E}>3.000 \mathrm{~lx}$ & Excede a faixa útil & $\begin{array}{c}\text { Excessiva, e pode produzir desconforto visual ou } \\
\text { térmico, ou ambos. }\end{array}$ \\
\hline
\end{tabular}

Fonte: adaptado de Mardaljevic et al. (2011). 
No artigo que define a IULN não se encontram menções a requisitos de quantidade de pontos de análise (NABIL; MARDALJEVIC, 2006). Entretanto, em algumas aplicações realizadas para atestar a sua eficácia esses autores utilizaram no máximo um ponto por metro quadrado. Os autores indicaram ainda que 0 estudo deveria ser anualizado, mas deixam a quantidade de dias e horas de análise a critério do projetista, que deve considerar os períodos de utilização do ambiente.

As normas NBR 15215-4 (ABNT, 2005c) - para luz natural - e a ISO/CIE 8995-1 (ABNT, 2013b) - para luz artificial - propõem o cálculo da quantidade de pontos de análise baseado nas dimensões do ambiente, mas apontam que o valor pode ser aumentado para melhor caracterização do ambiente. A norma de desempenho das edificações residenciais NBR 15575 (ABNT, 2013a) exige que o estudo seja feito em um ponto no centro do ambiente, em apenas quatro momentos ao longo do ano, em dois dias e em dois horários.

Ao tratar da quantidade de pontos necessária para o cálculo da ALN, Reinhart, Mardaljevic e Rogers (2006) definem que a malha de pontos deverá ter distância máxima de $0,5 \mathrm{~m}$ entre os pontos e que densidades maiores podem ser utilizadas em casos particulares. Apontam, ainda, que devem ser utilizadas todas as horas diárias de sol, mesmo que o ambiente não seja usado durante todo o período. Em casos particulares, outros intervalos podem ser utilizados. Autores como Dogan e Park (2018) trabalharam com essa variação temporal e perceberam que há significativa influência no resultado, quando a quantidade de horas de cálculo é alterada.

A LM 83-12 (ILLUMINATING..., 2012) descreve de forma objetiva os requisitos de horas e dias do ano para os índices ESA e ALNe que se realizará em intervalos de uma hora, das $8 \mathrm{~h}$ às $18 \mathrm{~h}$, horário legal, durante todo o ano, totalizando 3.650 horas. Define, ainda, que os pontos devem ter distância máxima de 0,60 m entre si e 0,30 m das paredes. Essa distância máxima foi definida por conseguir "atender adequadamente todas as possibilidades de área de trabalho” (IES, 2012). Os normativos citados adotam como altura do plano de trabalho: 0,75 m (ABNT, 2013a; 2013b), 0,76 m (ILLUMINATING..., 2012) e 0,80 m (REINHART; MARDALJEVIC; ROGERS, 2006).

Nesse contexto, faz-se necessário avaliar qual a influência dos parâmetros de cálculo escolhidos para o processamento computacional nos resultados obtidos e, consequentemente, na avaliação do desempenho luminoso de ambientes, utilizando os índices previamente descritos.
Este artigo tem como objetivo determinar a influência do tamanho da malha de pontos nos índices de desempenho luminoso: Em, U, ALN, ALNc, ALNe, IULN e ESA.

\section{Método}

O método utilizado busca avaliar a relação entre os índices de desempenho da luz natural e sua relação com a definição da malha de pontos de cálculo da iluminância. Os procedimentos e ferramentas utilizadas foram divididos em sete etapas descritas na Figura 2.

As dimensões e características dos materiais do ambiente foram definidas com base em trabalhos que avaliam a disponibilidade de luz natural no clima tropical (LARANJA, 2010; LIMA, 2015; MANHAS, 2016; CARVALHO, 2018; GUIDI et al., 2018; DIAS et al., 2018). O modelo adotado é um ambiente de 6,00 m x 6,00 m, com pé-direito de 3,00 m. A janela, em fita, com 1,00 m de pé-direito e 1,10 m de altura, conforme mostrado na Figura 3, sem obstruções externas. $\mathrm{O}$ vidro utilizado na janela é plano, comum, conforme Tregenza e Sharples (1993). As refletâncias das superfícies internas adotadas foram: 0,3 para o piso, 0,7 para as paredes internas e 0,8 para o teto.

Posteriormente foram escolhidos os índices de avaliação de desempenho da luz natural utilizados no estudo. A escolha se justifica nesta etapa metodológica tendo em vista que a determinação de alguns parâmetros de simulação, como malha de pontos e intervalo temporal, deve ser feita com base nos parâmetros requeridos para cada uma. Os índices escolhidos são apresentados no Quadro 2.

Em um estudo-piloto, com o mesmo ambiente e características dos materiais, foi verificado que a adoção do referencial de 300 lx para a ALNe seria facilmente atingido, fazendo com que os resultados do índice fossem pouco expressivos. Para minimizar tal efeito adotou-se o valor de 1000 lx, resultando em variações possíveis de serem analisadas estatisticamente. Os demais índices mantiveram seus valores referenciais.

As simulações ocorreram das 8h às $18 \mathrm{~h}$ (hora legal), no meio do intervalo, todos os dias do ano. Foi utilizado o céu com distribuição dinâmica de luminâncias (CDDL) para a cidade de Maceió. O CDDL escolhe um dos tipos de céu CIE (COMMISSION..., 2002), de acordo com sua probabilidade, no dia e na hora calculados, usando os estudos de Cabús (2002) e de Manhas (2016). O modelo de céu utilizado utiliza a subdivisão de 145 e 5.221 partes CIE (COMMISSION..., 2002) conforme Cabús (2002). 
Para todos os processamentos foi utilizado o mesmo modelo, variando apenas a malha de pontos, conforme o Quadro 3.

As malhas foram definidas variando-se a quantidade de pontos por eixo, de 2 a 15 . O ponto de análise está posicionado no centro de cada setor, de forma a preencher todo o plano de trabalho. O conjunto de malhas escolhidas contempla as previstas pelas normas NBR 15215-4 (ANBT, 2005c) e a IES LM-83-12 (ILLUMINATING..., 2012) e acrescenta outras com mais e menos pontos. A utilização de malhas diferentes das previstas em norma e a verificação da sua relação com os índices estudados é o principal objetivo do trabalho.

As duas orientações do modelo - sul e leste - foram escolhidas tendo em vista a trajetória solar para a cidade de Maceió, AL, cidade utilizada para a simulação. Para o modelo com janela voltada a sul o sol tende a iluminar diretamente uma porção menor do ambiente, mais próxima à janela, o que não acontece no modelo com janela orientada a leste, onde grande parte do ambiente, em metade do dia, recebe insolação.

A verificação da iluminância foi feita em um modelo computacional, utilizando o TropLux 7 (CABUS, 2005), software baseado nos métodos Monte Carlo, raio traçado e coeficientes de luz natural e largamente referenciado em trabalhos técnico-científicos (LARANJA, 2010; RAMOS; GHISI, 2010; BECKER et al., 2011; ELETROBRAS-PROCEL，2012; LAMBERTS; DUTRA; PEREIRA, 2014; HAREDY, 2016; WENA; HIYAMA; KOGANEI, 2017).

\section{Figura 2 - Fluxograma geral do método}

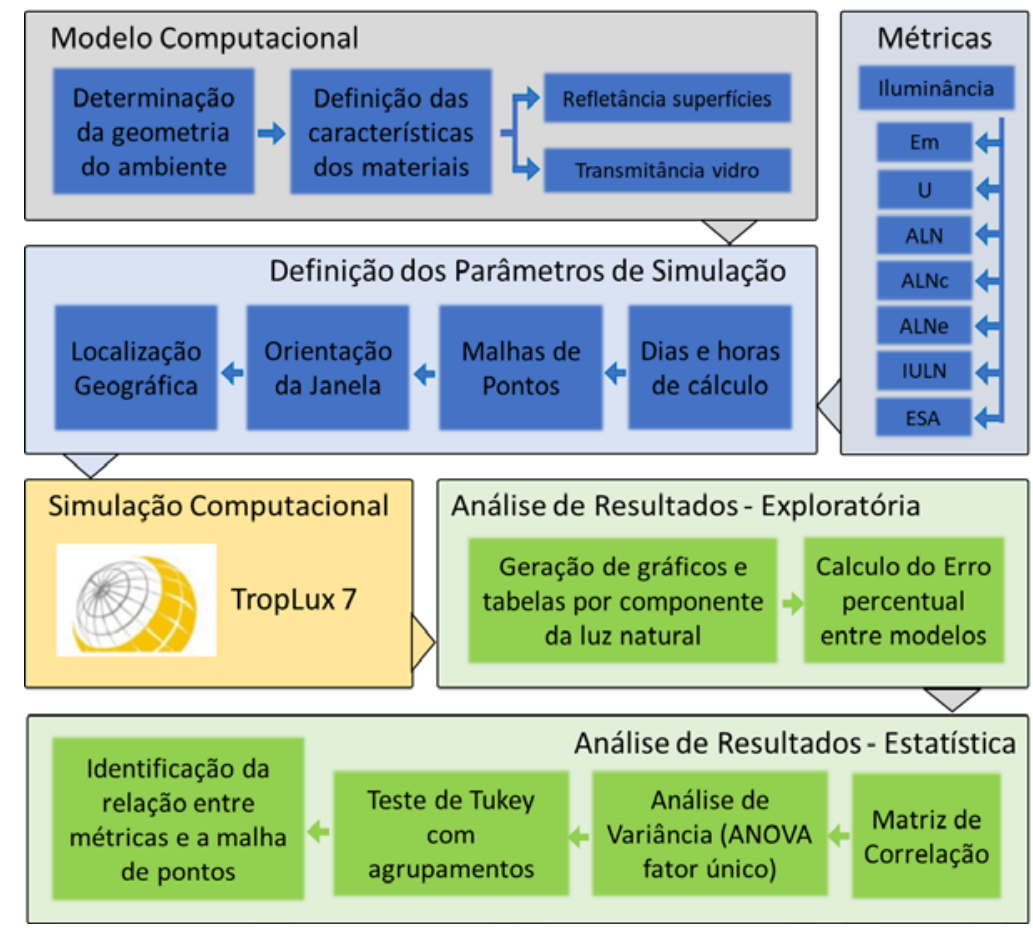

Figura 3 - Planta baixa e corte do ambiente utilizado nas simulações computacionais

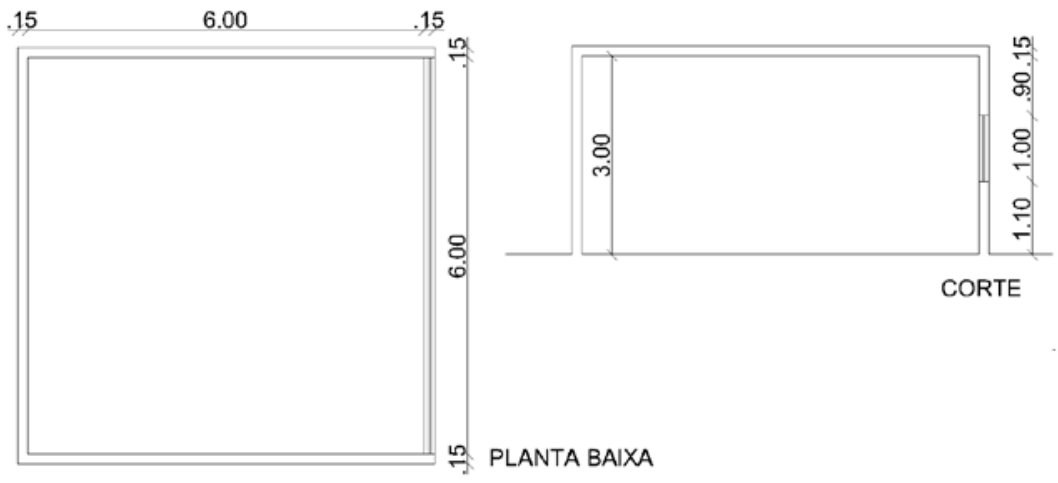


Quadro 2 - Resumo dos índices avaliados no trabalho, sua origem e método de cálculo

\begin{tabular}{|c|c|c|c|}
\hline Índice & Sigla & Referência & Forma de cálculo \\
\hline $\begin{array}{l}\text { Iluminância } \\
\text { média }\end{array}$ & Em & $\begin{array}{c}\text { (ANBT, 2005a, } \\
2005 b, 2005 c)\end{array}$ & $\begin{array}{l}\text { Calculada a iluminância em cada ponto da malha, } \\
\text { faz-se uma média de todos os valores no instante, } \\
\text { obtendo-se a iluminância média (Em) no plano de } \\
\text { trabalho. Para o estudo os dados foram agrupados de } \\
\text { duas formas: média anual (Ema) para a parte } \\
\text { exploratória e mensal para a estatística. }\end{array}$ \\
\hline $\begin{array}{l}\text { Uniformidade (da } \\
\text { iluminância) }\end{array}$ & $\mathrm{U}$ & ABNT (2013b) & $\begin{array}{l}\text { Razão entre a iluminância mínima e a iluminância } \\
\text { média em todos os pontos de análise em um instante. } \\
\text { Para o estudo os dados foram agrupados de duas } \\
\text { formas: média anual (Uma) para a parte exploratória } \\
\text { e mensal para a estatística. }\end{array}$ \\
\hline $\begin{array}{l}\text { Autonomia de } \\
\text { luz natural }\end{array}$ & ALN & $\begin{array}{c}\text { Reinhart e } \\
\text { Walkenhorst (2001) }\end{array}$ & $\begin{array}{l}\text { Percentual do conjunto de instantes (dias e horas) } \\
\text { em que um valor de iluminância é atingido no ponto } \\
\text { analisado. }\end{array}$ \\
\hline $\begin{array}{l}\text { Autonomia de } \\
\text { luz natural } \\
\text { contínua }\end{array}$ & ALNc & $\begin{array}{c}\text { Reinhart, } \\
\text { Mardaljevic e Rogers } \\
\text { (2006) }\end{array}$ & $\begin{array}{l}\text { Igual à ALN, entretanto o ponto recebe um crédito } \\
\text { parcial caso não atinja o limite definido, diretamente } \\
\text { proporcional à quão próximo ele chega do valor. }\end{array}$ \\
\hline $\begin{array}{l}\text { Autonomia de } \\
\text { luz natural } \\
\text { espacial }\end{array}$ & ALNe & $\begin{array}{l}\text { IES } \\
\text { (ILLUMINATING..., } \\
\text { 2012) }\end{array}$ & $\begin{array}{l}\text { Percentual da área do ambiente em que a ALN } \\
\text { ultrapassa um valor de iluminância determinado. O } \\
\text { normativo predefine um valor de } 300 \text { lx que pode } \\
\text { ser alterado a depender do uso do ambiente. }\end{array}$ \\
\hline $\begin{array}{l}\text { Iluminância útil } \\
\text { de luz natural }\end{array}$ & IULN & $\begin{array}{l}\text { Nabil e Mardaljevic } \\
\text { (2005) }\end{array}$ & $\begin{array}{l}\text { Classificação da iluminância em quatro grupos. } \\
\text { Cada grupo possui um limite inferior e superior de } \\
\text { iluminância. O índice é o percentual do ano de } \\
\text { ocorrências ao longo do em cada grupo. }\end{array}$ \\
\hline $\begin{array}{c}\text { Exposição solar } \\
\text { anual }\end{array}$ & ESA & $\begin{array}{c}\text { IES } \\
\text { (ILLUMINATING..., } \\
\text { 2012) }\end{array}$ & $\begin{array}{l}\text { Percentual da área do ambiente em que que a } \\
\text { componente de luz solar direta atinge ao menos } \\
1.000 \text { lx em mais de } 250 \text { horas por ano. }\end{array}$ \\
\hline
\end{tabular}

Quadro 3 - Disposição dos pontos analisados para cada modelo

\begin{tabular}{|c|c|c|c|c|c|c|}
\hline \multirow[b]{2}{*}{$\mathbf{n}^{0}$} & \multirow[b]{2}{*}{ Malha } & \multirow[b]{2}{*}{ Pontos } & \multirow[b]{2}{*}{$\begin{array}{c}\text { Distância } \\
\text { entre } \\
\text { pontos (m) }\end{array}$} & \multirow{2}{*}{$\begin{array}{l}\text { Distância entre } \\
\text { parede e ponto } \\
\text { (m) }\end{array}$} & \multicolumn{2}{|c|}{ Atende a: } \\
\hline & & & & & $\begin{array}{l}\text { NBR 15215-4 } \\
\text { (ANBT, 2005c) }\end{array}$ & $\begin{array}{c}\text { IES LM-83-12 } \\
\text { (ILLUMINATING..., } \\
\text { 2012) }\end{array}$ \\
\hline 1 & $2 \times 2$ & 4 & 3,000 & 1,500 & -1 & -1 \\
\hline 2 & $3 \times 3$ & 9 & 2,000 & 1,000 & -1 & -1 \\
\hline 3 & $4 \times 4$ & 16 & 1,500 & 0,725 & $-{ }^{1}$ & -1 \\
\hline 4 & $5 \times 5$ & 25 & 1,200 & 0,600 & Sim & -1 \\
\hline 5 & $6 \times 6$ & 36 & 1,000 & 0,500 & Sim & -1 \\
\hline 6 & $7 \times 7$ & 49 & 0,857 & 0,483 & -2 & -1 \\
\hline 7 & $8 \times 8$ & 64 & 0,750 & 0,375 & $-^{2}$ & -1 \\
\hline 8 & $9 \times 9$ & 81 & 0,667 & 0,333 & -2 & Sim \\
\hline 9 & $10 \times 10$ & 100 & 0,600 & 0,300 & -2 & Sim \\
\hline 10 & $11 \times 11$ & 121 & 0,545 & 0,273 & -2 & -2 \\
\hline 11 & $12 \times 12$ & 144 & 0,500 & 0,250 & -2 & -2 \\
\hline 12 & $13 \times 13$ & 169 & 0,461 & 0,231 & -2 & $-^{2}$ \\
\hline 13 & $14 \times 14$ & 196 & 0,429 & 0,215 & -2 & -2 \\
\hline 14 & $15 \times 15$ & 225 & 0,400 & 0,200 & $-^{2}$ & -2 \\
\hline
\end{tabular}

Nota: $-^{1}$ a dimensão da malha é menor que a prescrita pelo normativo; e $-^{2}$ a distância entre a o ponto e a parede é menor que o limite prescrito pelo normativo. 
A análise dos resultados foi realizada em duas etapas: exploratória e estatística. Na primeira os resultados foram avaliados de forma descritiva, sendo calculada a diferença percentual (DP) entre o modelo analisado e o de malha imediatamente inferior. Os índices iluminância e uniformidade tiveram suas componentes estudadas separadamente, sendo elas: luz do sol (SD), luz do sol refletida (SR), luz difusa de céu (CD) e luz difusa de céu refletida (CR), conforme Cabús (2005).

A análise estatística foi separada em duas etapas. $\mathrm{Na}$ primeira todos os resultados foram avaliados por meio de uma matriz de correlação e significância. $\mathrm{Na}$ segunda etapa foram utilizadas as ferramentas da análise de variância (One way ANOVA), utilizada para a comparação de múltiplas médias (MONTGOMERY; RUNGER, 2009). Para verificar as diferenças significantes entre as médias, como forma de determinar a partir de qual malha as médias dos índices são estatisticamente iguais, foi utilizado o teste de Tukey, que verifica quais as diferenças estatisticamente significantes entre as médias (CALLEGARI-JACQUES, 2003). O teste funciona como complementação da ANOVA, pois se nesta é verificado que as médias do conjunto são diferentes, o teste de Tukey indica quais são elas. Os cálculos foram feitos no software MINITAB versão $18.0^{1}$.

\section{Resultados e discussões}

Inicialmente foram avaliados os resultados da iluminância média anual (Ema) por componente. Também foi calculada a diferença percentual (DP) entre o valor obtido e o anterior. Na Figura 4 é possível verificar, graficamente, os resultados das simulações separados por componente.

Observa-se que a parcela de luz refletida, seja do sol ou do céu, demonstra tendência semelhante nas duas orientações estudadas, tendo comportamento uniforme, ao passo que a malha de pontos aumenta. Em malhas de 3 pontos por eixo (distância entre pontos de $1,5 \mathrm{~m}$ ) os resultados mostraram variação percentual menor que $5 \%$.

As malhas com 8, 9 e 11 pontos por eixo apresentaram ligeira mudança na tendência observada. Nessas malhas, para as demais componentes, também será observado o mesmo efeito, conforme demonstrado nas próximas análises. Em vista das alterações indicadas, faz-se necessária a avaliação aprofundada desses resultados. A componente de luz refletida apresentou variação significativa, ao passo que a quantidade de pontos na malha aumenta. Para a luz vinda do céu, de forma direta, malhas com mais de 4 pontos por eixo resultaram em diferenças percentuais menores que $5 \%$.

As componentes de luz direta, seja do sol ou do céu, apresentaram comportamento distinto, mantendo grande variação percentual com o aumento da quantidade de pontos. Para a luz direta do céu a orientação da janela foi determinante na ausência de variação nos resultados, o que não foi perceptível nas análises anteriores. Na orientação sul observase a estabilização da variação a partir da malha com três pontos por eixo, enquanto na orientação leste esse desempenho acontece a partir de 12 pontos por eixo. Quando analisada a componente de luz difusa de céu, observa-se grande alteração com o aumento da malha de pontos. Não é possível obter uma constância na diferença percentual para os modelos analisados, indicando que essa componente deverá precisar de mais pontos por eixo para manter um comportamento estável.

A síntese dos resultados pode ser observada no gráfico da iluminância global, que é a somatória de todas as componentes. Nesse quadro percebe-se a tendência de manutenção da diferença percentual menor que 5\% na malha com três pontos por eixo na orientação sul, e de seis pontos por eixo na orientação leste. Esse comportamento esconde o fato de que, analisadas separadamente, as componentes têm variações distintas e que para o cálculo da luz direta faz-se necessária a simulação de mais pontos por eixo a fim de se obterem resultados com grau de precisão aceitável.

A uniformidade média anual da iluminância também foi analisada em cada componente. Os resultados são apresentados na Figura 5. Observase que apenas a componente de luz do sol refletida apresenta diferença percentual maior que 5\% em grande parte dos cenários estudados. Para a componente de céu, direta ou refletida, independentemente da orientação, os valores da diferença percentual são menores que 5\% para malhas com mais de seis pontos por eixo.

Para o estudo da uniformidade da luz do sol, seja direta ou refletida, é preciso remeter-se diretamente ao comportamento da iluminância apresentado anteriormente. A luz solar direta apresenta grande variabilidade de Ema mesmo com o aumento da quantidade de pontos por eixo. Entretanto, não há variação da uniformidade na orientação sul, em todos os cenários estudados, o que reafirma a necessidade de uma malha mais refinada para a

10 Minitab é um software utilizado para estudos estatísticos desenvolvido em 1972 e continuado pela Minitab INC State College, PA EUA. 
determinação da iluminância quando da presença de insolação direta no espaço interno. Para orientação leste, observa-se estabilização da uniformidade, com seis pontos por eixo.

O comportamento distinto da uniformidade, nas duas orientações, justifica-se pelo fato de que na orientação sul a trajetória aparente do sol faz com que a região do plano de trabalho que recebe luz direta do sol esteja restrita a uma porção perto da janela, enquanto no ambiente com janela voltada a leste praticamente todo o plano de trabalho recebe luz solar direta, em algum instante do ano, na parte da manhã.

A avaliação da uniformidade média anual global $\left(U_{\mathrm{ma}}\right)$ permite observar que, para a orientação sul o ambiente tem variação percentual da uniformidade menor que $5 \%$, a partir da malha com cinco pontos por eixo, o que só vai acontecer na orientação leste a partir de dez pontos por eixo.
Para o estudo da IULN e da ALN e suas derivadas não foram feitas distinções entre as componentes, tendo em vista que a aplicabilidade dos conceitos só existe na análise da iluminância global. Com relação à ESA, conceitualmente, apenas a iluminância solar direta é estudada. Os resultados são apresentados na Figura 6.

Os gráficos da $A L N$ e da $A_{L} N_{c}$ apresentaram desvios muito pequenos em relação à média, e uma tendência rápida de estabilização dos seus valores. Essa tendência não é suficiente para afirmar que a quantidade de pontos no plano de trabalho não influencia o cálculo desses índices. A grande disponibilidade de luz natural encontrada na cidade utilizada nas simulações faz com que a quantidade de luz no ambiente seja suficiente para atingir o valor de referência na maioria dos instantes.

\section{Figura 4 - Gráficos de iluminância média anual (Ema) - o eixo horizontal representa a quantidade de pontos por eixo, o eixo vertical esquerdo representa a grandeza das barras, e o eixo vertical direito a diferença percentual representada pelas linhas}

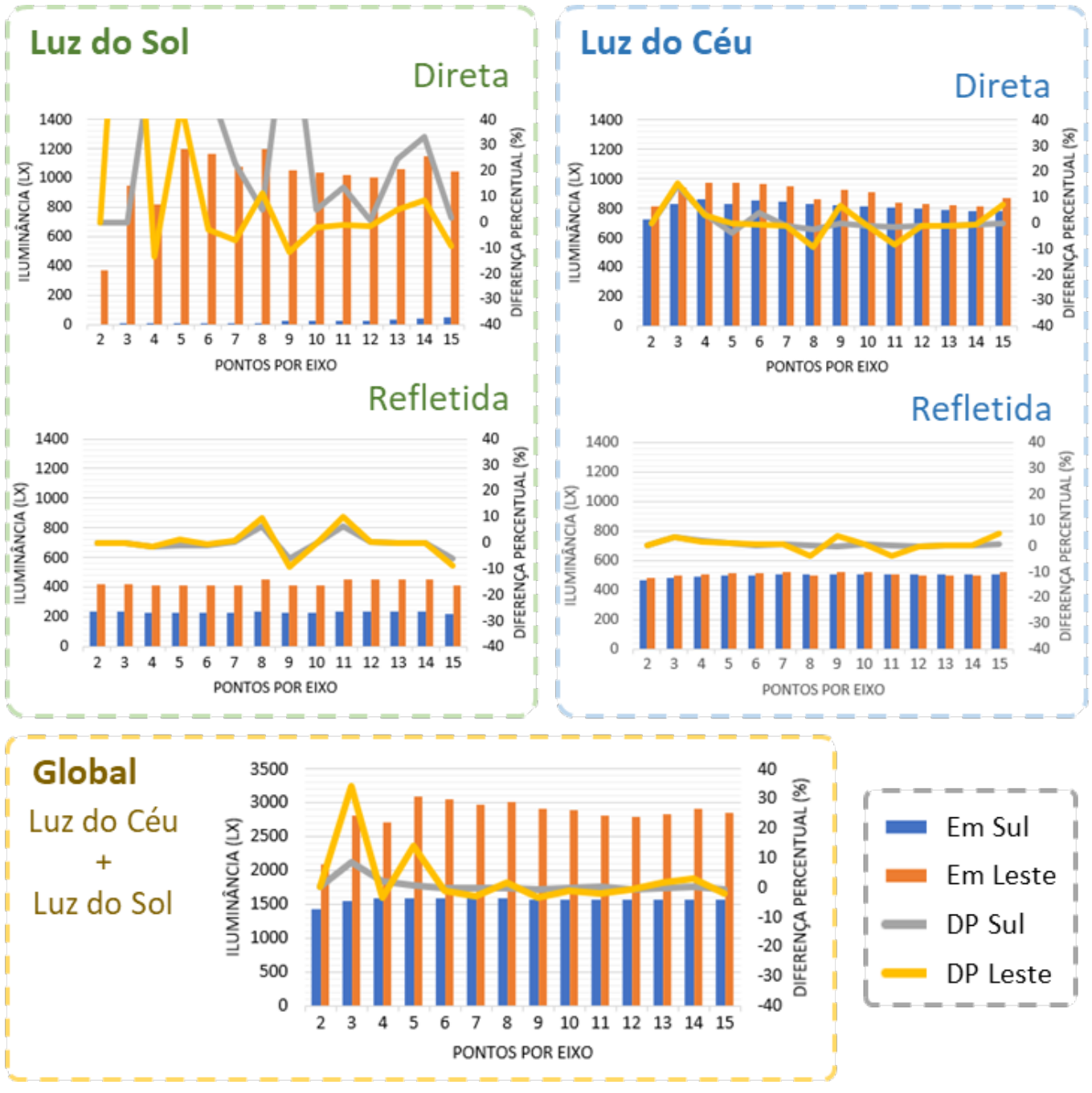


Figura 5 - Gráficos de uniformidade média anual (Uma) - o eixo horizontal representa a quantidade de pontos por eixo, o eixo vertical esquerdo representa a grandeza das barras, e o eixo vertical direito a diferença percentual representada pelas linhas

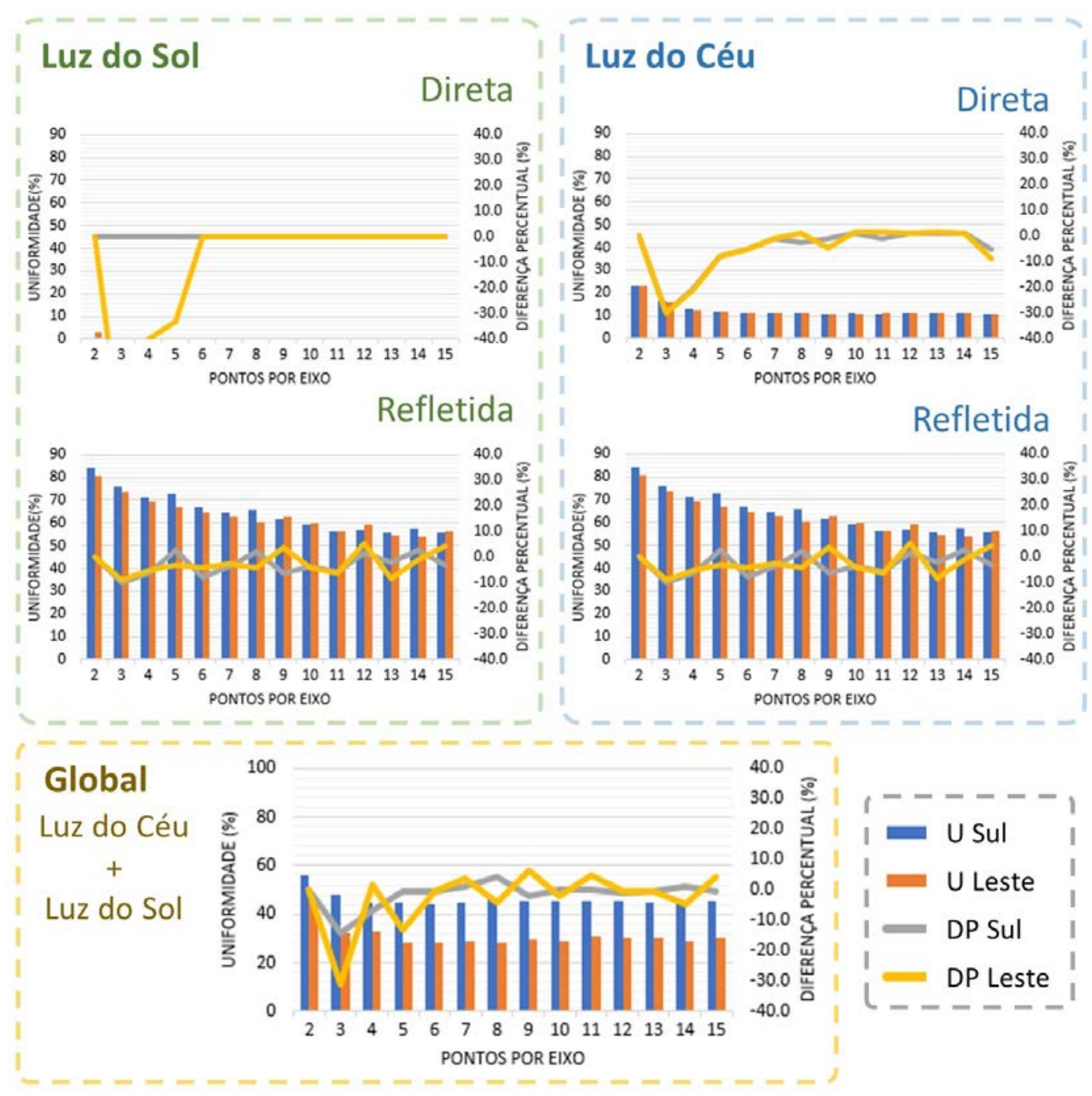


Figura 6 - Gráficos dos índices ALN, ALNc, ALNe, IULN e ESA - o eixo horizontal representa a quantidade de pontos por eixo, o vertical à esquerda representa a grandeza das barras, e o vertical à direita a diferença percentual representada pelas linhas

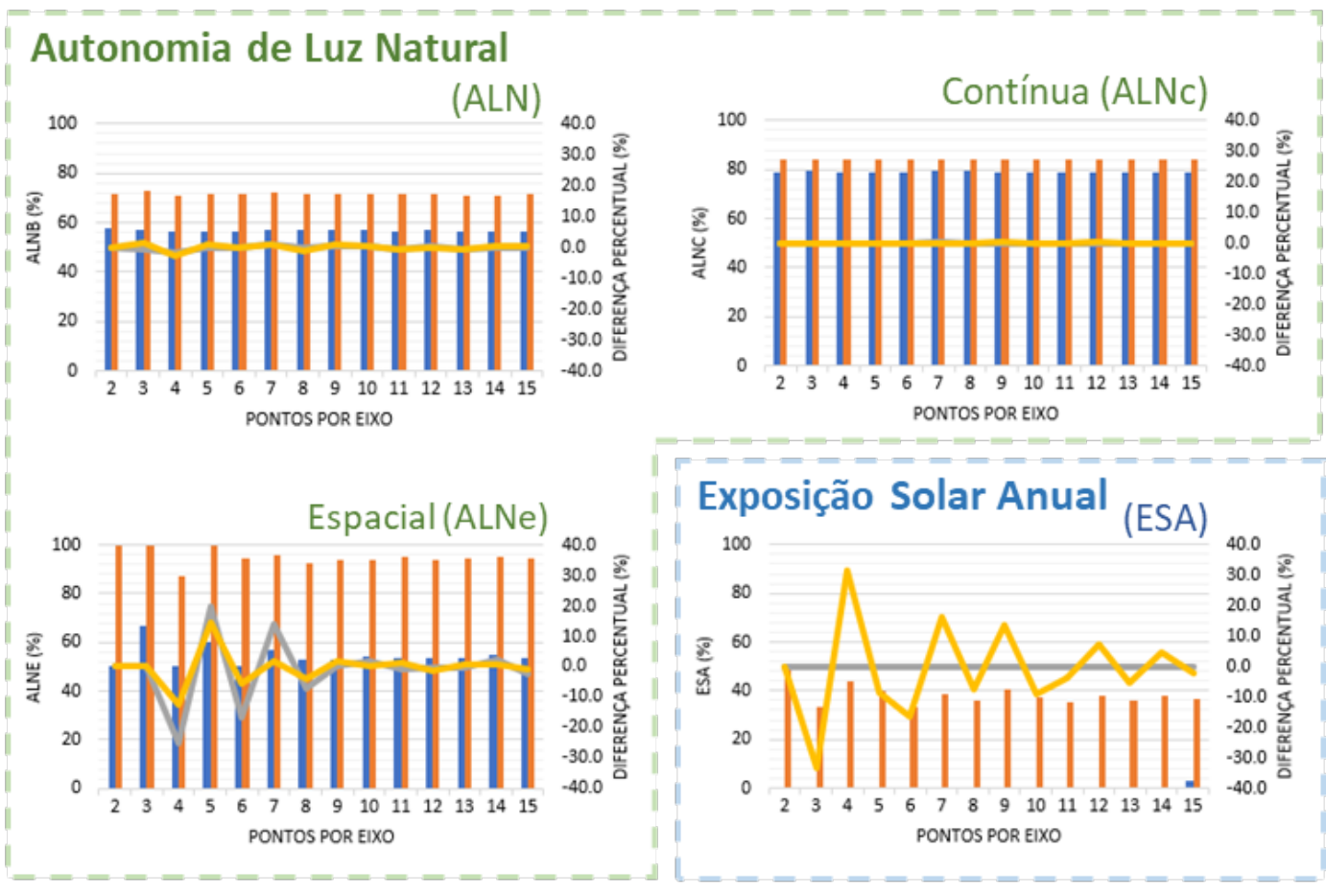

\section{Iluminância útil de Luz Natural}
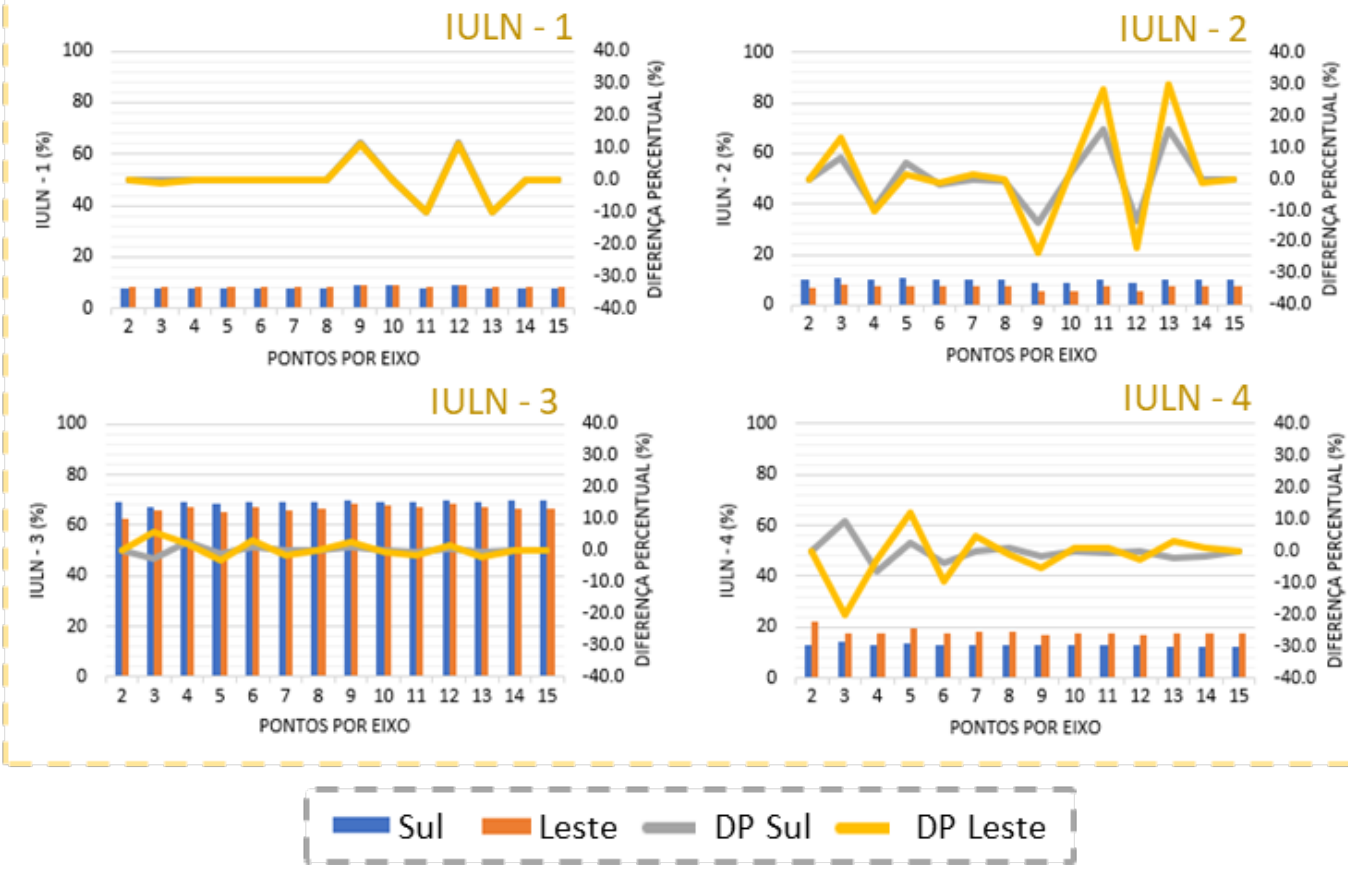

Quando a $\mathrm{ALN}_{\mathrm{e}}$ é avaliada ao longo de todo o ano sua variação se apresenta mais evidente. Para as duas orientações, as malhas menores, com até sete pontos por eixo, mostraram diferença percentual expressiva, tendendo a apresentar pequena diferença, a partir de oito pontos por eixo para ambas as orientações. Outro fator que apresentou grande variação foi a exposição anual solar. O gráfico mostra que apenas para os modelos com malhas maiores que 14 pontos por eixo os resultados tendem a ser constantes com a alteração da malha, ou são perceptíveis, como no caso da 
orientação sul, admitindo uma diferença de $4 \%$. Os gráficos de IULN apresentaram variação significativa da diferença percentual em malhas com mais pontos por eixo nas faixas 1 , 2 e 4, e não foi possível observar a estabilização na variação dos resultados. Já na faixa 3, a de maior interesse para aproveitamento da luz natural, observa-se uma diferença percentual menor que $10 \%$ a partir de malhas com oito pontos por eixo.

Na análise inicial foi possível perceber que certos parâmetros são mais facilmente influenciados pela quantidade de pontos do que outros, mas que todos, em algum grau, sofrem influência dessa escolha. Aqueles calculados de forma anualizada, como a ESA, ou a ALNe, apresentam maior relação com o número de pontos de forma mais evidente que os demais. A análise se aprofunda quando verificado que a quantidade de pontos está diretamente relacionada à distância entre eles. Os cenários podem ser divididos pela orientação, outro fator importante na decisão.

Na orientação sul - em que a trajetória solar aparente com relação à janela faz com que a insolação direta seja menor, como pode ser observado nos valores de ESA - há tendência de que seis pontos por eixo, o que corresponde a uma distância entre pontos de 1,00 m, apresente desvios menores que 1\% para Em e U. Para a ALN é preciso que haja mais de oito pontos por eixo, com distância entre pontos de no mínimo 0,75 m, para que a mesma diferença anterior seja obtida.

Na orientação leste, onde a trajetória solar faz com que haja maior entrada de luz solar direta, os resultados são diferentes, mas em todos é requerida uma quantidade de pontos por eixo menor para que a diferença percentual seja menor que $5 \%$. Na iluminância média são necessários ao menos oito pontos por eixo, com uma distância entre pontos de no mínimo $0,75 \mathrm{~m}$, para que a diferença se mantenha próxima aos $4 \%$. Já na ALNe são precisos 11 pontos por eixo, o que equivale a pontos distantes $0,54 \mathrm{~m}$ entre si.

Importante notar que a quantidade de luz do sol que entra nos ambientes influencia diretamente os índices de desempenho da iluminação natural e, consequentemente, a escolha da malha de pontos adotada. O fato indica que há uma relação entre a área da janela e sua posição na escolha da malha de pontos de análise. Os parâmetros de ALNe e ESA apresentaram maior sensibilidade à variação, necessitando de malhas com distância entre pontos menores que $0,54 \mathrm{~m}$, valor menor do que o prescrito pela IES LM-83-12 (ILLUMINATING..., 2012).

A fim de validar as inferências anteriores, os dados foram analisados utilizando ferramentas da estatística. Foi calculada a matriz de correlação, tendo como parâmetro de comparação a quantidade de pontos por eixo (PPE) e a distância entre pontos (DEP). Um resumo é apresentado na Tabela 1. Para o cálculo, foram tomados como referência dois parâmetros do modelo, a PPE e a DEP. É importante notar que a DEP mostrou correlações mais fortes do que a PPE, principalmente em orientações com maior entrada de sol ao longo do ano, indicando-a como um parâmetro relevante na escolha da malha de análise.

Para a $E_{m a}$, as componentes de luz difusa de céu e luz do sol refletida foram as que não alcançaram valores de correlação com DEP ou PPE altos. Entretanto, as componentes de luz do sol e luz difusa de céu refletida apresentaram correlações expressivas. A luz advinda do sol de forma direta apresentou forte correlação com a malha e significância em ambas as orientações. Já a correlação entre a luz difusa de céu refletida e os índices analisados foi determinante apenas para a janela voltada a sul.

Observa-se ainda que os destaques apresentados na Tabela 1 estão concentrados nas componentes de Ema e Uma, que, quando analisadas globalmente, independentemente da orientação, apresentaram os maiores valores de correlação e os menores de significância.

Os valores pouco expressivos encontrados nos demais índices de avaliação do desempenho da luz natural indicam que o cálculo está diretamente relacionado à correta definição dos parâmetros na iluminância e na uniformidade. Apenas a IULN nas faixas 3 e 4 apresentaram, mesmo que pequena, alguma correlação, para a orientação leste, com maior entrada de luz solar. Esse resultado, associado à maior presença de correlações significativas, indica que a orientação com maior entrada de luz solar direta é aquela em que a região de influência dos pontos de análise deve ser definida de forma mais cuidadosa, o que implica maior precisão dos resultados. Para a orientação sul, em que a entrada de luz solar é menor, ainda assim fazse necessário esse mesmo cuidado.

A não ocorrência de valores de ESA para a orientação sul em quase todos os modelos fez com que a correlação não se apresentasse significativa. Entretanto, na orientação leste, em que os valores de ESA variaram nos modelos, foi obtido um valor de correlação considerada significativa.

A matriz de correlação não consegue verificar as alterações dia a dia, já que trabalha apenas com o valor médio anual. Para tanto as análises de ANOVA foram realizadas utilizando dados mensais para a iluminância (E) e uniformidade (U). Um resumo dos resultados é apresentado na Tabela 2. 
Tabela 1 - Resumo da matriz de correlação entre a quantidade de pontos por eixo (PPE) e distância entre pontos (DEP) para os índices analisados nas duas orientações estudadas - as células destacadas em verde indicam as maiores correlações e as menores significâncias

\begin{tabular}{c|c|c|c|c|c|c|c|c}
\hline & \multicolumn{3}{|c|}{ Orientação sul } & \multicolumn{3}{c}{ Orientação leste } \\
\cline { 2 - 9 } & Correlação & Significância & \multicolumn{2}{c}{ Correlac̃o } & \multicolumn{2}{c}{ Significância } \\
\cline { 2 - 9 } & PPE & DEP & PPE & DEP & PPE & DEP & PPE & DEP \\
\hline$E_{\mathrm{ma}}-\mathrm{CD}$ & -0.26 & -0.24 & 0.37 & 0.40 & -0.55 & 0.14 & 0.04 & 0.63 \\
\hline $\mathrm{E}_{\mathrm{ma}}-\mathrm{CR}$ & 0.79 & -0.99 & 0.00 & 0.00 & 0.25 & -0.53 & 0.41 & 0.05 \\
\hline $\mathrm{E}_{\mathrm{ma}}-\mathrm{SD}$ & 0.98 & -0.77 & 0.00 & 0.00 & 0.47 & -0.80 & 0.09 & 0.00 \\
\hline $\mathrm{E}_{\mathrm{ma}}-\mathrm{SR}$ & 0.25 & -0.06 & 0.37 & 0.83 & 0.55 & -0.44 & 0.04 & 0.11 \\
\hline $\mathrm{E}_{\mathrm{ma}}-\mathrm{Global}$ & 0.35 & -0.76 & 0.23 & 0.00 & 0.36 & -0.72 & 0.25 & 0.00 \\
\hline $\mathrm{U}_{\mathrm{ma}}-\mathrm{CD}$ & -0.55 & 0.14 & 0.04 & 0.63 & -0.63 & 0.94 & 0.02 & 0.00 \\
\hline $\mathrm{U}_{\mathrm{ma}}-\mathrm{CR}$ & 0.24 & -0.53 & 0.41 & 0.05 & -0.87 & 0.91 & 0.00 & 0.00 \\
\hline $\mathrm{U}_{\mathrm{ma}}-\mathrm{SD}$ & 0.47 & -0.80 & 0.09 & 0.00 & -0.45 & 0.84 & 0.11 & 0.00 \\
\hline $\mathrm{U}_{\mathrm{ma}}-\mathrm{SR}$ & 0.55 & -0.44 & 0.04 & 0.11 & -0.93 & 0.96 & 0.00 & 0.00 \\
\hline $\mathrm{U}_{\mathrm{ma}}-\mathrm{Global}$ & 0.33 & -0.72 & 0.25 & 0.00 & -0.49 & 0.83 & 0.08 & 0.00 \\
\hline $\mathrm{ALN}$ & -0.47 & 0.73 & 0.09 & 0.00 & -0.10 & 0.32 & 0.74 & 0.26 \\
\hline ALN & -0.25 & 0.19 & 0.36 & 0.52 & -0.17 & 0.24 & 0.24 & 0.41 \\
\hline ALN & -0.23 & 0.11 & 0.42 & 0.70 & -0.31 & 0.45 & 0.29 & 0.11 \\
\hline IULN - 1 & 0.24 & -0.30 & 0.41 & 0.30 & 0.24 & -0.25 & 0.41 & 0.40 \\
\hline IULN - 2 & -0.33 & 0.37 & 0.25 & 0.20 & -0.33 & 0.31 & 0.25 & 0.29 \\
\hline IULN - 3 & 0.54 & -0.56 & 0.05 & 0.04 & 0.43 & -0.80 & 0.13 & 0.00 \\
\hline IULN - 4 & -0.57 & 0.54 & 0.03 & 0.05 & -0.55 & 0.78 & 0.04 & 0.00 \\
\hline ESA & 0.45 & -0.23 & 0.11 & 0.43 & -0.42 & 0.61 & 0.13 & 0.02 \\
\hline & & & & & & & &
\end{tabular}

Tabela 2 - Resumo dos resultados na ANOVA para iluminância (E) e uniformidade (U) - os resultados em destaque apontam a variável que não atende à hipótese testada; para a componente de luz do sol, por apresentar muitos valores zero, não foi possível o cálculo

\begin{tabular}{|c|c|c|c|c|c|c|c|c|c|c|c|}
\hline & \multirow{2}{*}{ Índice } & \multicolumn{7}{|c|}{ Entre grupos } & \multicolumn{3}{|c|}{ Dentro dos grupos } \\
\hline & & & SQ & gl & MQ & $\mathbf{F}$ & valor-P & F crítico & SQ & gl & MQ \\
\hline \multirow{8}{*}{$\begin{array}{l}\exists \\
\text { W }\end{array}$} & \multirow{4}{*}{$\mathrm{E}$} & $\mathrm{CD}$ & 222922 & 13 & 17148 & 0,2785 & 0,9939 & 1,7842 & $9 \mathrm{E}+06$ & 154 & 61573 \\
\hline & & CR & 21794 & 13 & 1676,5 & 0,135 & 0,9999 & 1,7842 & $2 \mathrm{E}+06$ & 154 & 12416 \\
\hline & & SD & 44578 & 13 & 3429,1 & 1,2781 & 0,2305 & 1,7788 & 450748 & 168 & 2683 \\
\hline & & SR & 1801.9 & 13 & 138,61 & 0,0222 & 1 & 1,7842 & 962463 & 154 & 6249,8 \\
\hline & \multirow{4}{*}{$\mathrm{U}$} & CD & 1905,5 & 13 & 146,57 & 38,771 & $7 E-42$ & 1,7842 & 582,19 & 154 & 3,7805 \\
\hline & & $\mathrm{CR}$ & 1614,8 & 13 & 124,22 & 130,13 & $6 \mathrm{E}-76$ & 1,7842 & 147 & 154 & 0,9546 \\
\hline & & SD & - & - & - & - & - & - & - & - & - \\
\hline & & SR & 11801 & 13 & 907,76 & 13,588 & $1 \mathrm{E}-19$ & 1,7842 & 10288 & 154 & 66,807 \\
\hline \multirow{8}{*}{ 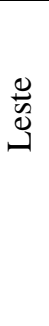 } & \multirow{4}{*}{$E$} & CD & 306478 & 13 & 23575 & 2,8272 & 0,0012 & 1,7842 & $1 \mathrm{E}+06$ & 154 & 8338,6 \\
\hline & & CR & 22012 & 13 & 1693,2 & 0,6866 & 0,7743 & 1,7842 & 379792 & 154 & 2466,2 \\
\hline & & SD & $6 \mathrm{E}+06$ & 13 & 476234 & 9,2746 & $6 \mathrm{E}-14$ & 1,7842 & $8 \mathrm{E}+06$ & 154 & 51348 \\
\hline & & SR & 522,14 & 13 & 40,165 & 0,0515 & 1 & 1,7842 & 120122 & 154 & 780,01 \\
\hline & \multirow{4}{*}{ U } & CD & 1816,5 & 13 & 139,73 & 5701,1 & 2E-199 & 1,7842 & 3,7744 & 154 & 0,0245 \\
\hline & & CR & 1528,8 & 13 & 117,6 & 257,85 & 2E-97 & 1,7842 & \begin{tabular}{|l|}
70,236 \\
\end{tabular} & 154 & 0,4561 \\
\hline & & SD & 56,382 & 13 & 4,3371 & 6,7757 & $3 E-10$ & 1,7842 & 98,575 & 154 & 0,6401 \\
\hline & & SR & 8970,1 & 13 & 690,01 & 132,53 & $2 E-76$ & 1,7842 & 801,76 & 154 & 5,2062 \\
\hline
\end{tabular}

Para as componentes em que o teste ANOVA indicou médias diferentes nos modelos foi realizado o teste de Tukey com agrupamentos, adotando uma confiança de $95 \%$. Os resultados dos agrupamentos são apresentados na Tabela 3 . O teste consegue definir a partir de que malha de pontos as médias podem ser consideradas iguais para a componente estudada.
Os resultados apresentados apontam definições importantes que ratificam as encontradas na análise exploratória inicial. Para a janela orientada a sul as alterações de iluminância não foram grandes o suficiente para que os resultados da análise estatística apontassem relação entre a iluminância e a malha de pontos. Entretanto, para a uniformidade, 
é possível definir diferenças nos resultados a partir da variação da malha.

O agrupamento de Tukey indica que, para estabilizar a média da uniformidade na janela orientada a sul, são necessários quatro pontos por eixo (distância entre pontos de $1,50 \mathrm{~m}$ ) na componente de luz difusa de céu (CD), e dez pontos por eixo (distância entre pontos de $0,60 \mathrm{~m}$ ) nas componentes de luz difusa de céu refletida (CR) e luz do sol refletida (SR). Essas duas componentes apresentaram eventos que alteraram os valores na malha de 15 pontos por eixo, em que houve uma redução ou um pico repentino. Os eventos indicam a necessidade de avaliações com malhas de mais pontos. Para essa orientação, a componente refletida apresentou necessidade de mais pontos que a componente direta.

Para a janela orientada a leste, a quantidade de pontos por eixo para estabilizar a média da iluminância varia entre oito, para a componente de luz difusa de céu, e sete para a de luz do sol. As demais componentes estabilizaram suas médias já na menor malha. Para a uniformidade, os resultados foram distintos em cada componente da luz, onde as malhas variam na quantidade de pontos por eixo de três (distância entre pontos de 2,00 m) na componente de luz do sol, passando por dez para as componentes de luz difusa de céu e refletido e indo até 13 na componente de luz do sol refletida (distância entre pontos de 0,46 m). Nas componentes de céu, aconteceram eventos de queda brusca nos valores para a malha de 15 pontos por eixo, o que reforça a necessidade de análises com malhas mais refinadas. A avaliação qualitativa da componente de luz do sol refletida mostrou grande variação e tendência de não estabilização da média, o que é ratificado pelos resultados da análise estatística.

A avaliação das duas formas de estudo, a exploratória e a estatística, permite concluir que as componentes apresentam comportamentos por vezes muito distintos entre si, entretanto é possível admitir alguns valores referenciais. Uma síntese dos resultados de iluminância e uniformidade é apresentada na Tabela 4 e, dos demais índices, na Tabela 5.

Tabela 3 - Resumo do teste de Tukey para iluminância (E) e uniformidade (U) apresentando os agrupamentos de cada componente - as barras mais grossas, destacadas entre as células, representam uma alteração no grupo entre elas; médias que não compartilham uma letra são consideradas significativamente diferentes

\begin{tabular}{|c|c|c|c|c|c|c|c|c|c|c|c|c|c|c|c|c|}
\hline & & & \multicolumn{14}{|c|}{ Pontos por eixo } \\
\hline & & Índice & 2 & 3 & 4 & 5 & 6 & 7 & 8 & 9 & 10 & 11 & 12 & 13 & 14 & 15 \\
\hline \multirow{3}{*}{$\vec{\Xi}$} & \multirow{3}{*}{$\mathrm{U}$} & $\mathrm{CD}$ & $\mathrm{A}$ & $\bar{B}$ & $\mathrm{C}$ & $\mathrm{C}$ & $\mathrm{C}$ & $\mathrm{C}$ & $\mathrm{C}$ & $\mathrm{C}$ & $\mathrm{C}$ & $\mathrm{C}$ & $\mathrm{C}$ & $\mathrm{C}$ & $\mathrm{C}$ & $\mathrm{C}$ \\
\hline & & CR & $\mathrm{A}$ & B & $\mathrm{B}$ & $\mathrm{C}$ & $\mathrm{C}$ & $\mathrm{CD}$ & $\mathrm{DE}$ & $\mathrm{DE}$ & $\mathrm{E}$ & $\mathrm{E}$ & $\mathrm{E}$ & $\mathrm{E}$ & $\mathrm{E}$ & $\mathrm{F}$ \\
\hline & & SR & $\mathrm{A}$ & $\mathrm{AB}$ & $\mathrm{BC}$ & $\mathrm{BC}$ & BCD & BCDE & BCDE & CDE & $\mathrm{DE}$ & $\mathrm{DE}$ & $\mathrm{DE}$ & $\mathrm{E}$ & $\mathrm{DE}$ & $\mathrm{E}$ \\
\hline \multirow{6}{*}{$\begin{array}{l}\stackrel{\Xi}{\tilde{\Xi}} \\
త\end{array}$} & \multirow{2}{*}{$\mathrm{E}$} & CD & $\mathrm{B}$ & $\mathrm{AB}$ & A & A & A & A & $\mathrm{AB}$ & $\mathrm{AB}$ & $\mathrm{AB}$ & $\mathrm{AB}$ & $\mathrm{AB}$ & $\mathrm{AB}$ & $\mathrm{AB}$ & $\mathrm{AB}$ \\
\hline & & SD & $\mathrm{C}$ & $\mathrm{AB}$ & $\mathrm{B}$ & A & A & $\mathrm{AB}$ & $\mathrm{AB}$ & $\mathrm{AB}$ & $\mathrm{AB}$ & $\mathrm{AB}$ & $\mathrm{AB}$ & $\mathrm{AB}$ & $\mathrm{AB}$ & $\mathrm{AB}$ \\
\hline & \multirow{4}{*}{$\mathrm{U}$} & $\mathrm{CD}$ & $\mathrm{A}$ & $\mathrm{B}$ & $\mathrm{C}$ & $\mathrm{D}$ & $\mathrm{E}$ & EF & $\mathrm{HI}$ & $\mathrm{HI}$ & GHI & IJ & GHI & FGH & EFG & $\mathrm{J}$ \\
\hline & & CR & $\mathrm{A}$ & $\mathrm{C}$ & B & $\mathrm{D}$ & $\mathrm{D}$ & $\mathrm{D}$ & $E$ & $\mathrm{E}$ & GHI & EF & FG & $\mathrm{EF}$ & EFG & $G$ \\
\hline & & SD & $\mathrm{A}$ & B & B & B & B & B & $B$ & B & B & B & B & B & B & B \\
\hline & & SR & A & B & $\mathrm{C}$ & $\mathrm{C}$ & $\mathrm{D}$ & $\mathrm{DE}$ & DEF & $\mathrm{DE}$ & EFG & $\mathrm{GH}$ & FGH & $\mathrm{H}$ & $\mathrm{H}$ & $\mathrm{H}$ \\
\hline
\end{tabular}

Tabela 4 - Síntese dos resultados obtidos para iluminância (E) e uniformidade (U) apresentando o número de pontos por eixo (PPE) e distância entre pontos (DEP) para cada índice estudado

\begin{tabular}{c|c|c|c|c|c|c|c|c|c|c|c}
\hline & \multirow{2}{*}{ Índice } & \multicolumn{4}{|c|}{ Iluminância (E) } & \multicolumn{4}{c}{ Uniformidade (U) } \\
\cline { 3 - 12 } & & CD & CR & SD & SR & GL & CD & CR & SD & SR & GL \\
\hline \multirow{2}{*}{ Sul } & PPE & 4 & 2 & $10^{(1)}$ & 2 & 4 & 7 & 10 & 2 & $5^{(2)}$ & 5 \\
& DEP (m) & 1,50 & 3,00 & 0,60 & 3,00 & 1,50 & 0,86 & 0,60 & 3,00 & 1,20 & 1,20 \\
\hline \multirow{2}{*}{ Leste } & PPE & $4{ }^{(3)}$ & 2 & $6^{(4)}$ & 2 & 4 & 6 & 6 & $6^{(5)}$ & $6^{(6)}$ & 4 \\
& DEP (m) & 1,50 & 3,00 & 1,00 & 3,00 & 1,50 & 1,00 & 1,00 & 1,00 & 1,00 & 1,50 \\
\hline
\end{tabular}

Nota: Legenda:

(1) Picos em 11, 13 e 14 PPE;

(2) Médias não constantes de Tukey até $13 \mathrm{PPE}$

(3) Valores intermediários pelo teste de Tukey a partir de $8 \mathrm{PPE}$;

(4) Valores intermediários pelo teste de Tukey a partir de 7 PPE;

(5) Teste de Tukey não conclusivo; e

(6) Muito variável pelo teste de Tukey, necessitando de mais avaliações. 
Tabela 5 - Síntese dos resultados obtidos para iluminância e uniformidade apresentando o número de pontos por eixo (PPE) e distância entre pontos (DEP) para cada índice estudado

\begin{tabular}{l|c|c|c|c|c|c|c|c|c}
\hline & \multirow{2}{*}{ Índice } & \multirow{2}{*}{ ALN } & \multirow{2}{*}{ ALNc } & \multirow{2}{*}{ ALNe } & \multicolumn{6}{|c|}{ IULN } & \multirow{2}{*}{ ESA } \\
\cline { 6 - 10 } & & & & Faixa 1 & Faixa 2 & Faixa 3 & Faixa 4 & \\
\hline \multirow{2}{*}{ Sul } & PPE & 2 & 2 & 9 & $-(1)$ & 6 & 2 & 5 & $-(1)$ \\
& DEP (m) & 3,00 & 3,00 & 0,67 & - & 1,00 & 3,00 & 1,20 & - \\
\hline \multirow{2}{*}{ Leste } & PPE & 2 & 2 & 7 & $-(1)$ & 5 & 4 & 7 & 11 \\
& DEP (m) & 3,00 & 3,00 & 0,86 & - & 1,20 & 1,50 & 0,86 & 0,55 \\
\hline
\end{tabular}

Nota: (1) inconclusivo com as análises realizadas.

Observa-se grande variabilidade nos resultados, entretanto é possível admitir que para o cálculo da iluminância, uma malha de quatro pontos por eixo é suficiente para obter resultados aceitáveis com margem de tolerância de 5\%. Já para atender aos critérios de uniformidade, é necessária uma malha com seis pontos por eixo. Importante observar que, mesmo com essas definições, a presença de variações, principalmente nas componentes refletida do céu e na luz do sol, faz com que a respectiva análise necessite de avaliação específica, a fim de validar os resultados obtidos.

Para os demais índices propostos, as malhas previstas pelas normas NBR 15215-4 (ANBT, 2005c) e para iluminância e uniformidade atendem bem à quantidade necessária de pontos. Apenas os índices de ALNe e ESA precisam ser avaliados com mais pontos, até 11 nesse último.

\section{Conclusão}

Os resultados apontam relações importantes entre os índices avaliados e a quantidade de pontos, ou a distância entre pontos, no plano de trabalho estudado. É possível afirmar que a distância entre pontos possui relação mais próxima aos índices de desempenho da luz natural que a quantidade de pontos na malha. Nesse sentido, a definição da quantidade de pontos em um plano de trabalho deverá considerar não apenas a quantidade, mas a distância entre eles. Ambientes com aberturas voltadas a orientações em que há entrada de luz solar direta em algum momento do dia apresentaram relação mais forte entre a malha de pontos e a variabilidade dos índices estudados. A pesquisa limitou-se a estudar a relação entre a malha e as métricas da luz natural, sem se aprofundar na forma de cálculo ou nos valores referenciais dos índices.

A avaliação separadamente dos índices que compõem a iluminância global aponta que há relações distintas entre eles e a malha de pontos. A luz vinda do sol, seja direta ou refletida, necessitou de mais pontos de análise para que os resultados se estabilizassem, ao contrário das componentes de céu, principalmente a direta, onde malhas menores apresentaram valores constantes.

Para atingir valores estáveis de iluminância global foi necessária malha de no mínimo 3x3 pontos no modelo com abertura voltada a sul, e de no mínimo 6x6 pontos no com abertura voltada a leste. Já a uniformidade apresenta mesmo comportamento para no mínimo $5 \times 5$ pontos na orientação sul e no mínimo 10x10 pontos na orientação leste. Observase que orientações com maior entrada de luz solar direta necessitam de malhas com mais pontos de análise.

A avaliação dos demais índices apontou para o cálculo da ALN, ALNc, ALNe e IULN uma malha de no mínimo 6x6 pontos, com distância entre pontos de $1,00 \mathrm{~m}$, e consegue obter resultados estatisticamente válidos, com pouca margem de diferença. A ALNe necessita de uma malha com mais pontos na orientação de maior entrada de luz solar direta. Importante notar que a ESA necessitou de uma malha de pontos com aproximadamente quatro vezes o necessário para os demais índices, com 11x11 pontos, ou distância entre pontos de $0,55 \mathrm{~m}$, indicando que quando há entrada de luz do sol, ou se deseja analisar especificamente esse índice, os pontos deverão ter no máximo essa distância entre eles.

Diante do modelo e das situações estudadas é possível afirmar que a distância entre os pontos deverá ser de, no máximo, 0,54 m, e que, em situações nas quais se deseje estudar a componente solar direta utilize-se a metade desse valor. Análises utilizando outros valores referenciais de ALN podem melhorar a avaliação desse índice, já que para o valor adotado os resultados não foram significantes. O valor corrobora o que autores como Reinhart, Mardaljevic e Rogers (2006) afirmam para a definição da ALN.

Observa-se que os índices de luz solar refletida, principalmente em orientações onde há maior entrada, precisam ser avaliados separadamente, pois sua variação nos modelos estudados foi expressiva. É possível, ainda, observar que se pode utilizar malhas diferentes para cada componente da luz natural, definindo malhas mais densas para a 
componente direta e menos densas para a componente difusa, como já apontam outros estudos (BREMBILLA; MARDALJEVIC; ANSELMO, 2015).

Quando comparadas as malhas mínimas identificadas no estudo com aquelas definidas em norma, observa-se que a norma brasileira relativa à avaliação da iluminação natural em edificações NBR 15215-4 (ABNT, 2005c) precisa ser revista para as novas métricas desenvolvidas.

Para o modelo estudado, com abertura lateral e sem obstruções externas, a malha definida pela norma brasileira, de $5 \times 5$ pontos, é inferior àquela necessária à definição de todos os índices estudados, como apresentado nos resultados. A ISO/CIE 8995-1 (ABNT, 2013b) traz uma formulação para iluminação artificial que se aproxima do prescrito pela IES LM-83-12 (IES, 2012), indicando a possibilidade de utilizar sua definição de malha de pontos para iluminação natural.

Os resultados apontam que a utilização de malhas diferentes no cálculo da iluminância, com mais pontos para a luz direta e menos para a luz difusa, pode ser estratégia importante na redução da demanda computacional, mantendo a precisão nos resultados.

\section{Referências}

ALBUQUERQUE, M. S. C.; AMORIM, C. N. D. Iluminação Natural: indicações de profundidadelimite de ambientes para iluminação natural no Regulamento Técnico da Qualidade do Nível de Eficiência Energética de Edifícios Residenciais RTQ-R. Ambiente Construído, Porto Alegre, v. 12, n. 2, p. 37-57, abr./jun. 2012.

ASSOCIAÇÃO BRASILEIRA DE NORMAS TÉCNICAS. NBR 15215-2: iluminação natural parte 2: procedimentos de cálculo para a estimativa da disponibilidade de luz natural. Rio de Janeiro, 2005a.

ASSOCIAÇÃO BRASILEIRA DE NORMAS TÉCNICAS. NBR 15215-3: iluminação natural parte 3: procedimento de cálculo para a determinação da iluminação natural em ambientes internos. Rio de Janeiro, 2005b.

ASSOCIAÇÃO BRASILEIRA DE NORMAS TÉCNICAS. NBR 15215-4: iluminação natural parte 4: verificação experimental das condições de iluminação interna de edificações: método de medição. Rio de Janeiro, 2005c.
ASSOCIAÇÃO BRASILEIRA DE NORMAS

TÉCNICAS. NBR 15575-1: edificações

habitacionais: desempenho: parte 1: requisitos gerais. Rio de Janeiro, 2013a.

ASSOCIAÇÃO BRASILEIRA DE NORMAS TÉCNICAS. ISO/CIE 8995-1: iluminação de ambientes de trabalho: parte 1: interior. Rio de Janeiro, 2013b.

ASSOCIATION SUISSE DES ELECTRICIENS. SN 418911: eclairage intérieur par la lumière du jour. Zurich, 1989.

BAKER, N.; FANCHIOTTI, A.; STEEMERS, K. Daylighting in Architecture: a european reference book. Londres: James \& James, 1993.

BECKER, A. et al. Diagnostico Casa Hacienda Quilapilún: Asesoría en eficiencia energética y calidad ambiental. In: UNIVERSIDAD

AUSTRAL DO CHILE (Org.). Dossier Laboratorio de Estudios de Sustentabilidad, Instituto de Arquitectura y Urbanismo. Valdivia: UAC, 2011.

BREMBILLA, E.; MARDALJEVIC, J.; ANSELMO, F. The Effect of the Analysis Grid on Daylight Simulations With Climate-Based Daylight Modelling. In: INTERNATIONAL COMMISSION ON ILLUMINATION, 28., Manchester, 2015. Proceedings... Manchester: CIE, 2015.

CABÚS, R. C. Tropical Daylighting: predicting sky types and interior illuminance in North-East Brazil. Sheffield, 2002. 288 f. Tese (Doutorado em Arquitetura) - University of Sheffield. Sheffield, 2002.

CABÚS, R. C. TropLux: um sotaque tropical na simulação da luz natural em edificações. In: ENCONTRO LATINO-AMERICANO, 4.; ENCONTRO NACIONAL SOBRE CONFORTO NO AMBIENTE CONSTRUÍDO, 8., Maceió, 2005. Anais... Porto Alegre: ANTAC, 2005.

CALIFORNIA ENERGY COMISSION. CEC 500-06-039: daylight metrics. São Francisco, 2012.

CALLEGARI-JACQUES, S. M. Bioestatística: princípios e aplicações. Porto Alegre: Artemed, 2003.

CARVALHO, M. L. S. Eficiência da Luz Solar Refletida e Desempenho de Dispositivos de Sombreamento: estudo para salas de aula na cidade de maceió. Maceió, 2018. 196 f. Tese (Doutorado em Engenharia Civil) - Programa de Pós-Graduação em Dinâmicas do Espaço Habitado, Universidade Federal de Alagoas, Maceió, 2018. 
CHARTERED INSTITUTION OF BUILDING SERVICES ENGINEERS. Lighting Guide 10: daylighting and Windows design. Londres: CIBSE, 1999.

\section{COMMISSION INTERNATIONALE DE}

L'ECLAIRAGE. CIE DS 011.2/E:2002: spatial distribution of daylight: CIE standard general sky. Viena, 2002.

DIAS, A. R. D. et al. Influência de Métricas Dinâmicas na Avaliação do Aproveitamento da Luz Natural em Clima Tropical. Ambiente Construído, Porto Alegre, v. 18, n. 3, p. 29-47, jul./set. 2018.

DOGAN, T; PARK, Y. A Critical Review of Daylighting Metrics for Residential Architecture and a New Metric for Cold and Temperate Climates. Lighting Research \& Technology, Londres, p. 1-25, fev. 2018.

ELETROBRAS-PROCEL. Manual para Aplicação do RTQ-R. Rio de Janeiro: Eletrobrás, 2012.

FERNANDEZ, P. M. E. Iluminación Natural Diseñada a Través de la Arquitectura. Sevilla, 2017. 634 f. Tese (Doutorado em Engenharia Civil) - Curso de Arquitectura, Universidad de Sevilla, Sevilla, 2017.

FONSECA, R. W, da; PEREIRA, F. O. R. Sequência Metodológica para a Estimativa da Iluminação Natural e Suas Implicações em Sistemas de Avaliação de Desempenho de Edificações. Ambiente Construído, Porto Alegre, v. 17, n. 1, p. 55-68, jan./mar. 2017.

FONTENELLE, M. R.; CLARO, A.; ARAÚJO, B. C. D. Influência de Elementos de Proteção Solar no Conforto Lumínico de Um Edifício Residencial Multifamiliar em Fortaleza - CE. In.: ENCONTRO NACIONAL DE CONFORTO NO AMBIENTE CONSTRUÍDO; ENCONTRO LATINO AMERICANO DE CONFORTO NO AMBIENTE CONSTRUÍDO, 11., Búzios, 2011. Anais... Rio de Janeiro: ANTAC, 2011.

GUIDI, C. R. et al. Influência dos Parâmetros Urbanísticos e da Topografia na Admissão da Luz Natural em Edifícios Residenciais. Ambiente Construído, Porto Alegre, v. 18, n. 3, p. 49-66, jul./set. 2018.

HAREDY, A. Simulation of Photovoltaic Airflow Windows for Indoor Thermal and Visual Comfort and Electricity Generation. Nottingham, 2016. 295 f. Tese (Doutorado em Arquitetura e Clima Urbano) - Departamento de Arquitetura e Clima Urbano, Universidade de Nottingham, Nottingham, 2016.
ILLUMINATING ENGINEERING SOCIETY OF NORTH AMERICA. LM-83-12: IES Spatial Daylight Autonomy (sDA) and Annual Sunlight Exposure (ASE). New York, 2012.

ILLUMINATING ENGINEERING SOCIETY OF NORTH AMERICA. The Lighting Handbook: reference \& applications. New York: Illuminating Engineering Society of North America, 2011.

INTERNATIONAL ORGANIZATION FOR STANDARDIZATION. 8995 CIE S 008/E: lighting of indoor work places. Genebra, 2002.

KLEINDIENST, S.; ANDERSEN, M.

Comprehensive Annual Daylight Design through a Goal-Based Approach. Building Research \& Information, v. 40, n. 2, p. 154-73, 2012

LAMBERTS, R.; DUTRA, L.; PEREIRA, F. Eficiência Energética na Arquitetura. 3. ed. Rio de Janeiro: Eletrobrás/PROCEL. 2014.

LARANJA, A. C. Parâmetros Urbanos e a Disponibilidade de Iluminação Natural no Ambiente Interno Rio de Janeiro. Rio de Janeiro, 2010. Tese (Doutorado em Arquitetura) Programa de Pós-Graduação em Arquitetura, Universidade Federal do Rio de Janeiro, Rio de Janeiro, 2010.

LIMA, R. C. Interferência do Entorno Construído na Disponibilidade de Luz Natural no Interior do Ambiente no Litoral Norte de Maceió - AL. Maceió, 2015. 147 f. Dissertação (Mestrado em Arquitetura e Urbanismo) Programa de Pós-Graduação em Dinâmicas do Espaço Habitado, Universidade Federal de Alagoas, Alagoas, 2015.

LITTLEFAIR, P. J.; AIZLEWOOD, M.; BIRTLES, A B. The Performance of Innovative Daylighting Systems. Renewable Energy, Londres, v. 5, n. 2, p. 920-934, jan. 1994.

MAIOLI, R. N.; ALVAREZ, C. E. de. Análise da Utilização de Prateleiras de Luz em Vitória-ES (Brasil). Hábitat Sustentable, Concepción, v. 3, n. 1, p. 37-46, jan. 2013.

MANGKUTO, R. A.; ROHMAH, M.; ASRI, A. D. Design Optimization for Window Size, Orientation, and Wall Reflectance With Regard to Various Daylight Metrics and Lighting Energy Demand: a case study of buildings in the tropics. Applied Energy, Londres, v. 164, p. 211-219, fev. 2016. 
MANHAS, M. P. G. Difusa ou Especular? Estudando o desempenho da prateleira de luz segundo a refletância de sua superfície. Maceió, 2016. 212 f. Dissertação (Mestrado em Arquitetura e Urbanismo) - Programa de Pós-Graduação em Dinâmicas do Espaço Habitado, Universidade Federal de Alagoas, Alagoas, 2016.

MARDALJEVIC, J. et al. Daylighting Metrics for Residential Buildings. In: SESSION OF THE CIE, 27., 2011, Sun City. Proceedings... Sun City: CIE, 2011.

MONTGOMERY, D. C.; RUNGER, G. C. Estatística Aplicada e Probabilidade para Engenheiros. 14. ed. Rio de Janeiro: LTC, 2009.

NABIL, A.; MARDALJEVIC, J. Useful Daylight Illuminance: a new paradigm for assessing daylight in buildings. Lighting Research \& Technology, Londres, v. 37, n. 1, p. 41-57, mar. 2005.

NABIL, A.; MARDALJEVIC, J. Useful Daylight Illuminances: a replacement for daylight factors. Energy and Buildings, Londres, v. 38, n. 7, p. 905-913, jul. 2006.

PEREIRA, D. C. L. Iluminação Natural em Edifícios de Escritório: metodologia para a avaliação do desempenho luminoso. São Paulo, 2017. 263 f. Tese (Doutorado em Engenharia Civil) - Faculdade de Arquitetura e Urbanismo, Universidade de São Paulo, São Paulo, 2017.

PHILLIPS, D. Natural Light in Architecture. Burlington: Architectural Press as Elsevier, 2004.
RAKHA, T.; NASSAR, K. Genetic Algorithms for Ceiling Form Optimization in Response to Daylight Levels. Renewable Energy, Londres, v. 36, n. 9, p. 2348-2356, set. 2011.

RAMOS, G.; GHISI, E. Analysis of Daylight Calculated Using the EnergyPlus Programme. Renewable and Sustainable Energy Reviews, v. 14, p. 1948-1958, 2010.

REINHART, C.; MARDALJEVIC, J.; ROGERS, Z. Dynamic Daylight Performance Metrics for Sustainable Building Design. Leukos, Ottawa, v. 3, n. 1, p. 7-31, jul. 2006.

REINHART, C.; WALKENHORST, O. Validation of Dynamic RADIANCE-Based Daylight Simulations for a Test Office With external BLINDS. Energy and Buildings, Freiburg, v. 33, n. 1, p. 683-697, jan. 2001.

SLATER, A. I; BOYCE, P. R. Illuminance Uniformity on Desks: Where is the limit? Lighting Research \& Technology, Londres, v. 4, n. 22, p. 165-174, 1990.

TREGENZA, P.; SHARPLES, S. Daylight Algorithms. Sheffield: Universidade de Sheffield, 1993.

WENA, L.; HIYAMA, K.; KOGANEI, M. A Method for Creating Maps of Recommended Window-to-Wall Ratios to Assign Appropriate Default Values in Design Performance Modeling: a case study of a typical office building in Japan. Energy \& Buildings, v. 145, p. 304-317, 2017.

Pedro Vitor Sousa Ribeiro

Faculdade de Arquitetura e Urbanismo | Universidade Federal de Alagoas | Campus A. C. Simões, Tabuleiro do Martins | Maceió - AL Brasil | CEP 57072-970 | Tel.: (82) 3214-1314 | E-mail: pedrovsribeiro@gmail.com

Ricardo Carvalho Cabús

Centro de Tecnologia | Universidade Federal de Alagoas | E-mail: r.cabus@ctec.ufal.br

Revista Ambiente Construído

Associação Nacional de Tecnologia do Ambiente Construído

Av. Osvaldo Aranha, 99 - 3o andar, Centro

Porto Alegre - RS - Brasil CEP 90035-190

Telefone: +55 (51) 3308-4084

Fax: +55 (51) 3308-4054

www. seer. ufrgs. br/ ambienteconstruido

E-mail: ambienteconstruido@ufrgs.br 